\title{
La signification du politique dans le rap
}

L'exemple du « rap de fils d'immigrés » (1997-2012)

The political meanings in rap music. The example of the "I'm an immigrant's son" (1997-2012)

\section{Pierre-Alain Clément}

\section{(2) OpenEdition}

\section{Journals}

\section{Édition électronique}

URL : http://journals.openedition.org/conflits/18972

DOI : $10.4000 /$ conflits. 18972

ISSN : $1777-5345$

\section{Éditeur :}

CECLS - Centre d'études sur les conflits - Liberté et sécurité, L'Harmattan

\section{Édition imprimée}

Date de publication : 30 juillet 2015

Pagination : 123-141

ISBN : 979-2-343-07175-6

ISSN : 1157-996X

\section{Référence électronique}

Pierre-Alain Clément, "La signification du politique dans le rap », Cultures \& Conflits [En ligne], 97 | printemps 2015, mis en ligne le 30 juillet 2016, consulté le 07 avril 2021. URL : http:// journals.openedition.org/conflits/18972; DOI : https://doi.org/10.4000/conflits.18972 


\section{La signification du politique dans le rap}

L'exemple du « rap de fils d'immigrés » (1997-2012)

\section{Pierre-Alain CLEMENT *}

Pierre-Alain Clément, diplômé de Sciences Po et de l'Université Lyon III, est doctorant à l'Université du Québec à Montréal. Il a notamment publié (avec Barthélémy Courmont) "When geopolitics meets the game industry. A study of Arabic video games and what they teach us", Hemispheres, 29/1, 2014. En plus de la culture populaire, il est spécialisé sur les questions de terrorisme (G.I. contre jihad : le match nul, Sainte-Foy, Presses universitaires du Québec, 2010) et de contre-terrorisme ("Le contre-terrorisme à l'ère multipolaire : consensus de façade, contestations infructueuses et déconvenues croissantes ", in Courmont B., Mottet E. (eds.), Repenser la multipolarité, Québec, Septentrion, 2013).

\section{Art engagé, rap et politique en France}

$\hat{A} \begin{aligned} & \text { mesure que déclinent, au tournant du Xxie siècle, les formes tradition- } \\ & \text { nelles d'engagement politique (adhésion aux partis, syndicalisation, intel- }\end{aligned}$ lectuels publics), la science politique s'est interrogée sur leurs mutations contemporaines, notamment les reconfigurations entre formes anciennes et nouvelles ${ }^{1}$. "À l'investissement total et durable de ceux qui font don de leur personne à leur cause, se substituerait un engagement "post-it", ponctuel et prêt à passer d'une cause à une autre, et auquel les individus désormais "distanciés" refuseraient de sacrifier leur vie privée et leur identité personnelle 2 ", soit un «investissement spontanéiste contre-culturel ${ }^{3}$ ». « L'atomisation et la privatisation 4 » de l'engagement sont dès lors apparues comme des changements centraux, par la mise à distance des grandes idéologies totalisantes au

*. L'auteur remercie les professeurs Anne-Marie D’Aoust, Francis Dupuis-Déri et Caroline Patsias ainsi que les deux évaluateurs pour leurs critiques et conseils. Cet article ne serait pas ce qu'il est sans la patience, l'exigence et la bienveillance de Clara Egger.

1. Perrineau P., «Les renouveaux de l'action politique », Vingtième siècle revue d'bistoire, 60, 1998, pp. 112-117 ; Muxel A., «La participation politique des jeunes : soubresauts, fractures et ajustements ", Revue française de science politique, 52/5-6, 2002, pp. 521-544.

2. Mathieu L., Comment lutter?, Paris, Textuel, 2004, p. 84.

3. Fillieule O., «Propositions pour une analyse processuelle de l'engagement individuel. Post scriptum », Revue française de science politique, 51/1, 2001, p. 210.

4. Perrineau P., op. cit., p. 116. 
profit d'engagements limités dans l'ambition, le temps et l'espace. Dans l'art, l'engagement est de plus en plus défini et traité de manière personnelle, focalisé sur l'action locale et limitée, quand ce n'est pas l'idée même d'engagement qui est disqualifiée ${ }^{5}$.

Déterminer la manière dont des artistes s’engagent nécessite de définir le politique à partir d'une définition qui inclue des formes non traditionnelles de participation politique. Dans une perspective qui combine constructivisme (importance des idées) et matérialisme (importance des structures) sur les causes de désaffection politique, $\mathrm{Hay}^{6}$ définit le politique comme la capacité d'agentivité (agency) et de délibération dans des situations de vrais choix collectifs ou sociaux. Selon cette définition, des activités très variées relèvent de la participation politique, de la parole à l'action, potentiellement violente, en passant par des formes de non-participation (boycott, abstention électorale ${ }^{7}$ ) ou des conversations « de tous les jours ", du moment qu'elles servent à exprimer « de l'exaspération, de la frustration, de l'irritation ou même de l'approbation envers les processus politiques formels ${ }^{8} »$. Dans cette perspective, $s$ 'approprier et alimenter le débat public, y compris en dehors de la sphère publique, est un acte politique.

Avec cette définition, l'œuvre d'art peut être comprise comme produisant ou participant à une situation politique. Si une œuvre comporte un message, surtout s'il est explicitement politique, les acteurs sont amenés à réfléchir à la situation collective décrite par l'œuvre, à en discuter, voire à agir dessus. La chanson francophone engagée utilise ce ressort et possède une tradition de contestation. Dans cet univers, le rap occupe une place unique. Musicalement apparenté au jazz et à la soul, il s'en distingue comme "première musique sans musicien professionnel », et il valorise, avec le punk et le rock (alternatif) l'autoproduction ${ }^{9}$ et le « faites-le vous-même » 10 . Comme le reggae, le rap fait valoir le «droit d'expression de catégories de populations habituellement exclues des forums publics et des arènes politiques ${ }^{11}$ ». Comme le rock, il représente une musique volontiers jugée comme contestataire ${ }^{12}$. Mais contrairement à ces genres, le rap possède un texte « long et détaillé », avec des cou-

5. Lafargue de Grangeneuve L., «L'ambivalence des usages politiques de l'art - Action publique et culture hip-hop dans la métropole bordelaise ", Revue française de science politique, 56/3, 2006, pp. 457-477 ; Bizzoni L., Prévost-Thomas C. (eds.), La Chanson francophone engagée, Montréal, Triptyque, 2008, pp. 10-11.

6. Hay C., Why we hate politics, Cambridge, Polity, 2007, p. 65.

7. La Rumeur revendique son abstentionnisme (voir Binet S., Mortaigne V., «Il n'y a pas plus politique que La Rumeur », Le Monde, 19 avril 2012.)

8. Hay C., op. cit., p. 75

9. Barrio S., Sociologie du rap français : état des lieux (2000-2006), thèse de doctorat en sociologie, 2007, pp. 244, 305.

10. Traïni C., La Musique en colère, Paris, Presses de Sciences Po, 2008, p. 47.

11. Ibid.

12. Cependant, à l'origine, le rap est simplement une musique festive, comme le montre la devise lancée par le précurseur Afrika Bambaataa : «Peace, love, unity and having fun ». 
plets «trois à cinq fois plus longs » que ceux de la variété et du rock et ses dérivés ${ }^{13}$. La révolte du rock est essentiellement celle de jeunes issus des «classes moyennes supérieures » dans un « conflit générationnel » quand celle du rap provient de jeunes exclus, notamment en raison de leurs origines, d'une société à laquelle « ils veulent fondamentalement appartenir ${ }^{14}$ ». En termes de consommation, le rap représente « un puissant repoussoir 15 ", deuxième genre qui ne plaît pas après le « métal / hard rock 16 ». C'est pourtant un genre fortement consommé, suivant un clivage générationnel (et genré) : premier genre musical préféré chez les 15-19 ans et les hommes de 15-30 ans.

Cet article veut comprendre les modalités politiques du rap, genre populaire mais clivant prisé par des populations exclues des processus politiques traditionnels. Il se focalise sur des artistes qui s'identifient et sont identifiés par les observateurs (journalistes, universitaires) comme politiques. Les artistes retenus sont le groupe La Rumeur et Casey, artistes les plus prolifiques ${ }^{17}$ et reconnus ${ }^{18}$ de ce qu'Hamé, de La Rumeur, a décrit comme le « rap de fils d'immigrés 19 » (R.F.I.). Ces artistes sont choisis pour trois raisons. D'abord, comme le suggère cette appellation, les rappeurs qui s'en revendiquent se disent politiques. Par exemple, Ekoué de La Rumeur : « Dans le rap français, et même dans les musiques actuelles, il n'y a pas plus politique que La Rumeur. [En effet,] notre musique est censurée. En quinze ans, je n'ai jamais vu sur ma feuille de SACEM un passage sur une radio nationale. Cela étant, nous avons choisi de tourner le dos à Skyrock 20 ». Ensuite, parce que le message politique du R.F.I., sans être l'apanage de ces artistes, est spécifique : il traite des dominations dont souffrent les Français d'origine africaine dans une perspective historique qui remonte à l'esclavage et la colonisation mais sans appeler, contrairement à d'autres rappeurs politisés, à l'engagement politique (vote, travail associatif, etc.) Enfin, l'originalité principale du R.F.I. est son refus d'intégrer l'industrie du rap et la dénonciation de, ou l'absence de collaboration avec, la quasi-totalité des autres rappeurs.

13. Barrio S., op. cit., pp. 79, 81.

14. Souchard M., "La différence rap », in Darré A., Musique et politique, Presses universitaires de Rennes, 1996, pp. 260, 262.

15. Pecqueux A., Le Rap, Paris, Le Cavalier bleu, 2009, p. 37.

16. Donnat O., Les Pratiques culturelles des Français à l'ère numérique, enquête 2008, Paris, ministère de la Culture et de la Communication / La Découverte, 2009.

17. Ils ont produit dix-sept albums sur les trente rattachés à cette appellation.

18. Leurs produits bénéficient d'un succès critique (deux chansons figurent dans le classement du magazine The Source de 2004 et quatre dans celui du webzine l'Abcdrduson de 2009) et commercial (quatre albums de La Rumeur et trois de Casey figurent dans le classement des deux cents disques les plus vendus pendant au moins une semaine) supérieur à celui des quatre autres rappeurs de l'appellation (Sheryo, Navea, B. James et Prodige).

19. On l'abrège par unique souci de commodité en R.F.I.

20. Binet S., Mortaigne V., op. cit. 


\section{Spécificité du R.F.I. dans le paysage français}

La Rumeur et Casey, figures les plus prolifiques et reconnues de la dizaine d'artistes se revendiquant du R.F.I., sont issus d'anciennes colonies françaises : le Togo (Ekoué), l'Algérie (Hamé), la Guadeloupe (Le Bavar) et la Martinique (Casey). La dimension politique est un fondement du R.F.I. : La Rumeur et Casey ont explicité dès le début leur positionnement dans l'industrie du rap, comme le relèvent les présentations médiatiques ${ }^{21}$ et universitaires ${ }^{22}$.

L'appellation R.F.I. est avant tout une marque de distinction. Elle ne s'inscrit pas dans les taxinomies populaires : rap « conscient » (pour les textes politisés ou engagés socialement), rap « de rue » (pour les textes retraçant les "galères » de la vie quotidienne dans la banlieue ou le quartier populaire), rap " égotrip » (pour les exercices de style consistant à se vanter exagérément en rabaissant les autres rappeurs), rap festif. Par opposition, c'est un endonyme qui s'oppose de manière explicite à l'appellation « rap français » et qui porte un message tiers-mondiste : comme le dit Hamé, c'est un « rap de fils de travailleur [domination socio-économique] immigré [domination postcolonialiste] ${ }^{23}$ ». En faisant de l'extranéité à la France une référence fondamentale, le R.F.I. est le parangon du rap français vu comme « produit musical postcolonial 24 ». La subalternité ressentie des Français d'origine africaine 25 s'inscrit dans une subalternité née de l'histoire de l'immigration et de la colonisation. De l'entre-deux-guerres aux années 1960, l'immigration maghrébine est économique, ouvrière et agricole, et se mettent en place les conditions de sa mise à l'écart spatiale, économique et identitaire. En effet, la prise en charge par l'État des immigrés décolonisés, par le «contrôle tant en matière de déplacement que d'hébergement » dans les foyers de travailleurs, reproduit le modèle colonial du « regroupement ségrégatif de civils $26 »$. La restriction de l'immigration à partir des années 1960, axée sur le regroupement familial, n'est pas

21. «Rares sont les groupes de rap français qui affirment une identité politique et une conscience sociale sans être en contradiction avec un matérialisme banlieusard purulent. » (Cachin O., Hip Hop : l'authentique histoire en 101 disques essentiels, Paris, Scali, 2007, p. 281.)

22. La Rumeur appartient aux groupes ayant un « discours social et politique fort 》 (Molinero S., Les Publics du rap, Paris, L'Harmattann, 2009, p. 139), avec des «thématiques post-coloniales violemment politiques » (Pecqueux A., Le Rap, op. cit., p. 60). La Rumeur vise à « remettre en cause la violence d'un discours politique dominant qui ne peut se défaire d'un atavisme colonialiste où l'indigène, l'immigré et le Français fils d'immigrés d'origine africaine ne font qu'un et représentent toujours, comme au passé, un groupe social considéré comme illégitime. » (Berrada T., « La Rumeur et son rap : emploi de la rumeur comme fonction discursive contestataire », Contemporary French Civilization, 37/1, 2012, p. 63.)

23. «On m'a demandé un jour comment je qualifiais mon rap, et j’ai répondu que c'était du rap de fils d'immigré, de fils de travailleur immigré. » (Tévanian P., « Entretien avec Mohamed Bourokba, dit Hamé, du groupe La Rumeur », Les Mots sont importants, 30 juin 2010, http://lmsi.net/L-intrus-du-groupe-1, Page consultée le 2 juin 2015.)

24. Béru L., « Le Rap français, un produit musical postcolonial ? », Volume, 6/1-2, 2009, pp. 6179 .

25. Kepel G., Banliene de la République. Société, politique et religion à Clichy-sous-Bois et Montfermeil, Paris, Gallimard, 2012.

26. Bernardot M., "Camps d'étrangers, foyers de travailleurs, centres d'expulsion : les lieux communs de l'immigré décolonisé », Cultures et conflits, 69, 2008, pp. 60-61. 
accompagnée d'une politique à « visée assimilatrice 27 » des immigrés dont on n’envisage plus le retour au pays. La question de l'intégration des populations immigrées émerge à partir des années 1980, et l'on assiste depuis la fin des années 1990 à la montée des thèmes conservateurs et répressifs, voire xénophobes, y compris à gauche ${ }^{28}$, accentuée par des difficultés économiques persistantes : chômage de masse, faible croissance, creusement des inégalités. La dénonciation de la mise à l'écart et du rôle de bouc émissaire des (descendants d') immigrés se fait explicite chez des rappeurs disant : « je suis qu'un passeport sous scellé / voilà mon seul rapport à la citoyenneté » $\left(2009 \mathrm{~b}{ }^{29}\right)$ et « aux darons sous litron, gosses illettrés / considérés comme en trop, triés au droit d'entrée / tous à errer en rond aux environs de Paris / là où les rues sont barrées dès qu'un grand baron se marie » (2010).

Mais dire que le R.F.I. est politique ne fait que soulever d'autres questions : depuis 1990, les médias ont fréquemment choisi de voir dans les rappeurs des «symptômes de problèmes publics » spécifiques aux banlieues, dans une forme "d'exotisme anthropologique 30 ». Cela fait dire à Hammou : "Qu'est-ce que désigner quelque chose ou quelqu'un comme “politique”, côté commentateur, et qu'est-ce qu'être dit “politique”, côté rappeurs / rappeuses ? 31 ». Or, toute catégorisation est le résultat d'un rapport de force. Pour Hammou, la politisation du rap procède de deux dynamiques : l'appropriation du rap comme moyen d'exprimer les effets concrets de la paupérisation des quartiers populaires ; et l'interprétation systématiquement politisée du rap par les médias ${ }^{32}$. Dans ce cadre, "l'argumentation devient le critère d'évaluation du rap dès lors qu'il y est question de politique. (...) Or, faire du rap une argumentation ne peut que conduire à une déception dont l'intensité varie en fonction des espoirs, des attentes que les auteurs placent dans cette “argumentation politique" 33 ». Producteur d'un objet culturel populaire, le rappeur politique semble condamné à susciter auprès des représentants de la culture dominante des réactions oscillant entre paternalisme enthousiaste, déçu ou légitimiste. La conclusion de Pecqueux est sans appel : «Évaluer le rap comme argumentation politique aboutit souvent au déni de la moindre dimension politique au rap. (...) Si les rappeurs développent pour ces auteurs une conscience sociale évidente, ce qui est de leur compétence, ce n'est pas le cas pour la politique : leur argumentation est insuffisante 34 ». Cette logique ravale le rap politique au rang de divertissement populaire inoffensif.

27. Idem, p. 67.

28. Mucchielli L., La Frénésie sécuritaire - Retour à l'ordre et nouveau contrôle social, Paris, La Découverte, 2008.

29. Toutes les citations du corpus sont identifiées par l'année de publication de l'album qui les contient. Voir la discographie en fin d'article.

30. Hammou K., Une histoire du rap en France, Paris, La Découverte, 2012, pp. 74-80.

31. Hammou, K., «Le rap est-il politique ? Question ouverte », Sur un son rap, 8 février 2010 (http://surunsonrap.hypotheses.org/1762, Page consultée le 2 juin 2015).

32. Hammou K., Une histoire du rap en France, op. cit., p. 136-141.

33. Pecqueux A., Voix du rap. Essai de sociologie de l'action musicale, Paris, L'Harmattan, 2007, p. 36. 
Fréquente dans l'analyse du rap français, cette logique oscille entre deux biais normatifs : le populisme et le misérabilisme ${ }^{35}$. Le point commun de ces biais consiste à systématiquement parler de l'objet de culture populaire en fonction de la culture légitime. En axant notre étude sur le R.F.I. compris comme élément du rap français, il ne sera pas systématiquement comparé à la culture légitime. Plus généralement, il est nécessaire de prendre le texte au sérieux sans oublier qu'il s'agit d'art, afin d'éviter ce que Fabiani appelle le "pathos de l'intellectuel légitime qui fait de l'immigré des quartiers le dernier héros culturel $36 »$.

\section{Méthodologie et cadre de recherche}

Un objet de culture populaire peut être analysé sous quatre angles : le contexte, le texte, la réception, le recodage. Mais une « étude de la longueur d'un article ne peut sélectionner qu'une ou deux de ces approches 37 ». En l'occurrence, l'analyse se concentre sur «la chanson en tant que texte » exprimant des idées, sans préjuger de la réception ${ }^{38}$ ni de la performance. Ce choix permet de traiter en profondeur l'ensemble d'un corpus, de bâtir des catégories robustes et d'illustrer les résultats par des citations représentatives. Afin de limiter au maximum la subjectivité, on se borne à l'intentionnalité explicite du texte. Cette restriction est particulièrement adaptée au rap, dont les « chansons sont d'abord discours et messages », le rythme musical venant « en soutien du sens plutôt qu'il ne l'oblitère 39 », le texte, " prise de parole individuelle adressée à une entité collective (...) prenant le dessus sur la musique 40 ».

Le corpus est composé de chansons. Cet article ne retient que les albums produits aux noms de Casey, de La Rumeur et des rappeurs du groupe. Il écarte les titres courts sur les compilations et les invitations par d'autres artistes. On obtient un corpus cohérent et exhaustif, composé de 180 chansons (appr. 73000 mots). Les paroles ont été retranscrites manuellement et passées au crible d'une analyse de contenu ${ }^{41}$.

34. Idem, p. 37.

35. Passeron J.-C., Grignon C., Le Savant et le Populaire. Misérabilisme et populisme en sociologie et en littérature, Paris, Gallimard et Le Seuil, 1989.

36. Cité dans Pecqueux A., "C’est quand il y en a beaucoup qu'il y a des problèmes », Sur un son rap, 21 mai 2014 (http://surunsonrap.hypotheses.org/2989, Page consultée le 2 juin 2015).

37. Hinds H., "A holistic approach to the study of popular culture: context, text, audience, and recoding”, in Hinds H., Motz M., Nelson A., Popular culture theory and methodology, Madison, University of Wisconsin Press, 2006, p. 164.

38. Frith S., Performing rites. On the value of popular music, Cambridge, Harvard University Press, 1996, p. 164.

39. Souchard M., op. cit., p. 260.

40. Barrio S., op. cit., pp. 66, 81.

41. Bardin L., L'Analyse de contenu, Paris, Presses universitaires de France, 1980 (2e éd.) ; Ghiglione R., Matalon B., Bacri N., Les Dires analysés. L'analyse propositionnelle du discours, Paris, Presses universitaires de Vincennes, 1985. 
L'analyse de contenu se fonde sur Garand et Hayward 42 qui distinguent six catégories dans une chanson contestataire : auprès d'un public, le sujet dénonce un tort causé par un anti-sujet à une victime et demande réparation. Une étude exploratoire permet d'éliminer trois catégories moins pertinentes : le public, « concerné » 43 mais aussi élargi ; les sujets, composés de La Rumeur et Casey ; et la victime : les sujets et par extension, les Français d'origine africaine et les banlieusards. En analysant les deux chansons contenant l'expression « rap de fils d'immigrés », on obtient les sous-catégories suivantes.

Trois anti-sujets émergent. Des institutions répressives, le " 9 place Beauvau» (2004), mais aussi des «institutions bourgeoises» (2007a). Au niveau professionnel, sont condamnés les « vendus devenus trop riches propriétaires du micro » (2007a).

Ces anti-sujets sont auteurs de torts, comme les «brutales méthodes» de la police, «nos séquelles » de l'histoire et les « interdits d'antenne » des radios (2004). Sont aussi dénoncées les violences symboliques de ces dominations, comme « la censure » et les "grandes fêtes foraines républicaines » chargées de mettre de l'ordre dans « nos bons vieux trous à rats » (2004).

Des réparations sont revendiquées, comme l'insoumission des «non-alignés, qualifiés d'infâmes diffamateurs » et des minoritaires « absents au banc des priorités » (2004). Le R.F.I. est "étranger à leurs codes ", allusion au " code pénal à tranche dorée » (2004) et antimétabole du Code des étrangers, créé en 2004. Au niveau professionnel, le R.F.I. « balaye les prestations bidons / enraye les disques moisis » (2007a) caractéristiques des «sales groupes» et de « ce dancefloor de salopes du rap et du R'n'B non stop ${ }^{44}$ » (2004). Le R.F.I. propose de sortir de la complaisance dans le statut passif de victime. C'est aux dominés d'être les agents de leur revalorisation, en tant que producteurs d'un art mineur, c'est-à-dire d'art " d'une minorité fait dans une langue majeure 45 » : Ékoué «s'autoproclame en mission jusqu'aux abords de ton coffre-fort » et « renvoie les pitres à leur cahier toujours avec le sourire » (2007a).

Pour opérationnaliser l'analyse, le contenu a été divisé en deux. D’un côté, l'étude exploratoire, inductive, fait ressortir un contenu "politique », exprimant les anti-sujets, les torts et les réparations. De l'autre, le contenu " autre", comprenant tout le reste. Suite à cette étude par codage ouvert, on postule que le R.F.I. identifie un ordre dominant dont il dénonce les torts et pour lesquels il réclame réparation.

42. Garand D., Hayward A. (eds.), États du polémique, Montréal, Nota Bene, 1998.

43. Pecqueux A., Voix du rap, op. cit., pp. 33-34.

44. Référence au slogan de Skyrock.

45. Béthune C., «Le hip hop : une expression mineure », Volume, 8/2, 2011, p. 162. 
On applique ensuite une démarche déductive, par un codage fermé créant des sous-catégories ${ }^{46}$, composées de trois formes de domination : socio-économique, postcolonialiste (comprise comme la perpétuation de la pensée coloniale après la fin du colonialisme) et professionnelle. Cette démarche ne considère donc que les formes de domination qui apparaissent explicitement dans le corpus, et ne se penche pas sur les dominations qu'il ignore ou encourage.

L'intégralité du contenu est codé par (portions de) phrases ${ }^{47}$. La congruence entre nos catégories de contenu politique et notre cadre théorique garantit deux exigences fondamentales d'une analyse de contenu : l'homogénéité et la pertinence des catégories ${ }^{48}$. Le contenu autre, non traité, est codé de manière ouverte, résultant en sept catégories. Le Tableau 1 détaille le codage des catégories.

\section{Description des résultats}

On tire trois enseignements des Tableaux 2 et suivants ${ }^{49}$. Premièrement, on remarque la force du contenu politique (66\%, minimum par album : $45 \%$ ). Sur les 17 albums, seulement deux ont moins de 50\% de contenu politique. Euvre artistique, une large partie du texte relève du contenu autre, composé notamment de figures imposées dans le rap : narration personnelle sans portée politique (13\%), égotrip $(8 \%)$ et interpellation directe $(2 \%)$.

Ensuite, on note la force de la dénonciation des torts. Les deux catégories les plus fortes sont la domination socio-économique et la domination postcoloniale (32\%). L'une de ces deux catégories est la plus importante dans neufs albums.

Enfin, le poids des réparations professionnelles, quatrième catégorie la plus importante $(9 \%)$, catégorie de réparation la plus fréquente dans huit albums. Dans le sous-total industrie du rap (12\%), le contraste est frappant entre les torts (1\%) et les réparations $(9 \%)$. Deux raisons peuvent expliquer ce contraste. D’abord une raison générale : dans un milieu marqué par la compétition et le défi comme le rap ${ }^{50}$, il est risqué de dire que d'autres rappeurs dominent. Ensuite, une raison particulière : La Rumeur et Casey ont obtenu un certain succès professionnel sans «se compromettre » avec les acteurs

46. Lewins A., Silver C., Using software in qualitative research: a step-by-step guide, Londres, Sage, 2007.

47. Ghiglione R., Matalon B., Bacri N., op. cit., pp. 18-24.

48. Idem, p. 121.

49. Calcul : nombre de caractères divisé par nombre total de caractères (incluant les co-occurrences). Pourcentages arrondis à l'unité. En gras, la catégorie la plus importante de chaque album. Sous-total autres en gras si inférieur à 50\%. Analyse effectuée avec le logiciel d'analyse qualitative Weft QDA.

50. Miliani H., "Culture planétaire et identités frontalières. À propos du rap en Algérie ", Cahiers d'études africaines, 168, 2002, p. 767. 
Tableau 1 : Catégories de codage

\begin{tabular}{|c|c|c|c|}
\hline \multicolumn{2}{|l|}{ Catégories } & \multirow{2}{*}{$\begin{array}{l}\text { Description } \\
\text { Acteurs producteurs (dirigeants poli- } \\
\text { tiques/éco.) et coercitifs (police, justice) } \\
\text { de la domination socio-économique }\end{array}$} & \multirow{2}{*}{$\begin{array}{l}\text { Citation type } \\
\text { "ceux qui s'abreuvent du sang et de la sueur de } \\
\text { l'homme " } \\
\text { "ta police, ta justice et ton fisc / et tes partis } \\
\text { gauchistes aux soi-disant prises de risque » }\end{array}$} \\
\hline $\begin{array}{l}\text { I. Anti- } \\
\text { sujets }\end{array}$ & $\begin{array}{l}\text { 1. Dominant } \\
\text { socio- } \\
\text { économique }\end{array}$ & & \\
\hline & $\begin{array}{l}\text { 2. Dominant } \\
\text { postcolonialiste }\end{array}$ & $\begin{array}{l}\text { Acteurs producteurs (France raciste, } \\
\text { médias, école) et coercitifs (police, jus- } \\
\text { tice) de la domination postcolonialiste }\end{array}$ & $\begin{array}{l}\text { "la France et son passé de colon » } \\
\text { "vos livres d'histoire » } \\
\text { " ce grand pays de fachos et de flicaille» }\end{array}$ \\
\hline & $\begin{array}{l}\text { 3. Industrie } \\
\text { du rap }\end{array}$ & $\begin{array}{l}\text { Acteurs dominants de l'industrie du rap } \\
\text { (éditeurs, radios, rappeurs à succès) }\end{array}$ & $\begin{array}{l}\text { "les autres trouducs / que les playlists des radios } \\
\text { ont transformés en eunuques" }\end{array}$ \\
\hline \multirow[t]{3}{*}{ II. Torts } & $\begin{array}{l}\text { 1. Domination } \\
\text { socio- } \\
\text { économique }\end{array}$ & $\begin{array}{l}\text { - Subalternité socio-économique, ségré- } \\
\text { gation spatiale, confrontation judiciaire } \\
\text { - Violence symbolique légitimant ces do- } \\
\text { minations }\end{array}$ & $\begin{array}{l}\text { - « autant de familles entassées en déchus } \\
\text { quartiers ouvriers » } \\
\text { - « la meilleure des polices / c'est quand les pau- } \\
\text { vres / savent rester à leur place / sans besoin de } \\
\text { les matraquer / de leur coudre la mâchoire / ou } \\
\text { de les mettre au cachot» }\end{array}$ \\
\hline & $\begin{array}{l}\text { 2. Domination } \\
\text { postcolonialiste }\end{array}$ & $\begin{array}{l}\text { - Perpétuation de la hiérarchie identi- } \\
\text { taire en défaveur des Français d'origine } \\
\text { africaine } \\
\text { - Violence symbolique légitimant ces do- } \\
\text { minations }\end{array}$ & $\begin{array}{l}\text { - "la ségrégation bat à son plein / des traduc- } \\
\text { tions truquées / intégration sinon rien » } \\
\text { - "le maintien dans l'ignorance, la désillusion / } \\
\text { devient la forme d'agression la moins efficace du } \\
\text { colon » }\end{array}$ \\
\hline & $\begin{array}{l}\text { 3. Domination } \\
\text { professionnelle }\end{array}$ & $\begin{array}{l}\text { - Injonction à devenir un rappeur con- } \\
\text { sensuel } \\
\text { - Violence symbolique légitimant ces do- } \\
\text { minations }\end{array}$ & $\begin{array}{l}\text { - «c'est pas le genre de rap qu'ils veulent » } \\
\text { - " inexistant sur les ondes hertziennes / mais } \\
\text { gratifié par la profession à titre honorifique » }\end{array}$ \\
\hline \multirow[t]{3}{*}{$\begin{array}{l}\text { III. } \\
\text { Réparations }\end{array}$} & $\begin{array}{l}\text { 1. Sortir de } \\
\text { la domination } \\
\text { socio- } \\
\text { économique }\end{array}$ & $\begin{array}{l}\text { - Réussite professionnelle, victoire judi- } \\
\text { ciaire } \\
\text { - Réappropriation de la dignité de } \\
\text { minorité socio-économique }\end{array}$ & $\begin{array}{l}\text { - « un élément nocif qui fait du rap à but } \\
\text { lucratif » } \\
\text { - « la banlieue Nord mes frères / honneur à la } \\
\text { banlieue Nord» }\end{array}$ \\
\hline & $\begin{array}{l}\text { 2. Déconstruire } \\
\text { le postcolonial- } \\
\text { isme }\end{array}$ & $\begin{array}{l}\text { Réappropriation de la dignité de } \\
\text { minorité identitaire }\end{array}$ & 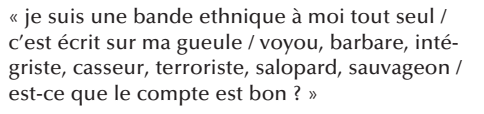 \\
\hline & $\begin{array}{l}\text { 3. Échapper à } \\
\text { l'industrie du rap }\end{array}$ & $\begin{array}{l}\text { Produire une œuvre légitime mais qui } \\
\text { ne se compromet pas avec l'industrie }\end{array}$ & $\begin{array}{l}\text { " nous sommes les premiers sur le rap fils de } \\
\text { pute / cette poignée de non-alignés qui com- } \\
\text { mencera par buter / rappeurs, DJ, et producteurs } \\
\text { zélés " }\end{array}$ \\
\hline \multirow[t]{7}{*}{ IV. Autres } & 1. Titres & Tous les titres des chansons & "Les Bronzés font du rap » \\
\hline & 2. Citations & $\begin{array}{l}\text { Toutes les citations de tiers (extraits } \\
\text { audio) dans les chansons }\end{array}$ & $\begin{array}{l}\text { "imaginez ce spectacle extraordinaire, dix vol- } \\
\text { cans à la fois crachant leur lave pour faire la } \\
\text { Martinique" }\end{array}$ \\
\hline & $\begin{array}{l}\text { 3. Adresse } \\
\text { à auditeur }\end{array}$ & Interpellations directes de l'auditeur & $\begin{array}{l}\text { "donc ce que je récite, je suis pas sûr que tu le } \\
\text { captes" }\end{array}$ \\
\hline & 4. Égotrip & $\begin{array}{l}\text { - Valorisation volontairement exagérée } \\
\text { de l'artiste et de ses compétences } \\
\text { - Interpellation aux amis } \\
\text { - Exclamations conventionnelles (nom } \\
\text { de l'artiste, année, etc.) }\end{array}$ & $\begin{array}{l}\text { - «Anfalsh et Zone libre c'est pas vraiment le son } \\
\text { des vacanciers / viens donne ta gorge qu'on } \\
\text { aiguise nos scalpels » } \\
\text { - " toi mon support si je vacille / Ekoué explique- } \\
\text { leur bien qu'on est une famille » } \\
\text { - « le B.A.V.A.R./ la R.U.M.E.U.R./ en 2-0-0-7» }\end{array}$ \\
\hline & $\begin{array}{l}\text { 5. Pensées } \\
\text { et histoires } \\
\text { personnelles }\end{array}$ & $\begin{array}{l}\text { Narration de réflexions introspectives et } \\
\text { de faits vécus ou imaginés sur des } \\
\text { thèmes variés sans connotation poli- } \\
\text { tique (famille, difficultés du quotidien, } \\
\text { soirées, etc.) }\end{array}$ & $\begin{array}{l}\text { "j'ai sorti les ciseaux de ma trousse avec la } \\
\text { frousse / et l'intention de leur faire mal à la sor- } \\
\text { tie de classe / je rumine, je ressasse et sans cesse } \\
\text { je repense / au seau d'eau dans ma face qui a tué } \\
\text { ma petite enfance" }\end{array}$ \\
\hline & $\begin{array}{l}\text { 6. Dénonciation } \\
\text { vague }\end{array}$ & $\begin{array}{l}\text { Dénonciation d'un tort ou réclamation } \\
\text { d'une réparation : } \\
\text { - sans qu'un auteur ou une victime soit } \\
\text { clairement identifiable ou ; } \\
\text { - lorsque la victime n'est pas un Français } \\
\text { d'origine africaine/banlieusard }\end{array}$ & $\begin{array}{l}\text { - «ceux qui nous empoisonnent l'existence par } \\
\text { fonction » } \\
\text { - « urgent, recherche troupeau de sans-papiers } \\
\text { parlant peu français / sous arrêt d'expulsion / } \\
\text { taillable et jetable, jetable et taillable pour } \\
\text { chantier / grand stade en construction » }\end{array}$ \\
\hline & $\begin{array}{l}\text { 7. Retour } \\
\text { aux sources }\end{array}$ & $\begin{array}{l}\text { Narration des pensées de l'artiste lors } \\
\text { d'un séjour dans le pays/la région d'orig- } \\
\text { ine et de ses traits caractéristiques }\end{array}$ & $\begin{array}{l}\text { " devant les cle-ons du bled devant la famille / } \\
\text { pas de hip-hop machin pas de pantalon aux } \\
\text { chevilles / c'est sûr le genre de truc qu'ils ne } \\
\text { conçoivent pas de rigolo / plutôt une baffe dans } \\
\text { la gueule et un aller Paris-Togo » }\end{array}$ \\
\hline
\end{tabular}


dominants de l'industrie du disque. Ils n'ont pas été diffusés par Skyrock et un seul des dix-sept albums du corpus (et même des trente albums du « rap de fils d'immigrés») a été édité par une major de l'édition musicale (2003 par EMI). De la sorte, le R.F.I. a réussi à ne pas se plier aux injonctions de l'industrie du rap.

L'analyse des sous-catégories montre les trois formes de l'ordre dominant dénoncé : la répression maintenant la domination socio-économique; la France «de l'apartheid » (2009b) et « son passé de colon » (2007b); et les rappeurs.

L'analyse des sous-catégories montre les formes de l'ordre dominant dénoncé. Quatre anti-sujets représentent les dominants socio-économiques : le juge, le nanti, le policier, le politicien. Les trois premiers font même l'objet de chansons entières $(2003,2006 a, 2009 a, 2010)$. Le politicien est dénoncé, de la manière la plus large (« ce système oppressant ») à la plus précise (Sarko, Claude Guéant). Les médias, les élections et l'école légitiment cette domination (« l'antenne qui informe / que les clichés sur nous sont bien réels et conformes », 2006a).

Six figures représentent les dominants postcolonialistes : la France, la Françafrique, la police, les médias, la justice et l'école. La France «machine à broyer nos traditions et cultures » (2003) est la principale accusée. L'extrême droite n'est pas la seule cible : « je ne cherche pas que les coupables capables de nous dire en face / qu'ils croient en l'inégalité des races / je cherche aussi leurs complices à décrypter leurs vices / partisans modérés d'une gestion policière. » (2007a). Le passé colonial et le présent néocolonial sont ensuite accusés : «l'aventure s'arrête là / Chirac, Foccart, Pasqua, Mitterrand (...) les suceurs de sang jurent fidélité / à Total, Accor, Bouygues, Bolloré » (2007b). Les médias et l'école sont à nouveau accusés de légitimer cette domination («les oublis dans les livres d'histoire, les pertes de mémoire», 1997).

Trois figures représentent les dominants professionnels, soit le diffuseur, l'éditeur et surtout le rappeur : «Et ils sont combien au bas mot / à s'embourgeoiser au micro / à s'écouter couler des crétineries » (2007a), « suceurs, zoophiles, consanguins, bienvenue chez les MC» (2008), « aux antipodes de ces groupes de merde qui ont déçu / et qui gèrent leur fin de carrière avec des godes dans le cul » (2003). Skyrock est la cible suivante : « radio de pointeur de gamines comme son P.D.-G. 51 » (idem), «putain d'opportuniste quasiesclavagiste / toi t'es qui pour me rayer de la playlist » (2006c), «j'encule Skyrock et tous ses animateurs ses programmateurs » (2008).

51. Pierre Bellanger a écopé de trois ans de prison avec sursis et 50000 euros d'amende pour corruption de mineur. 


\begin{tabular}{|c|c|c|c|c|c|c|c|c|c|c|c|c|c|c|c|c|c|c|}
\hline 馬 & \multicolumn{8}{|c|}{ 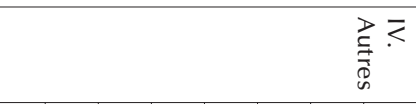 } & \multicolumn{3}{|c|}{ 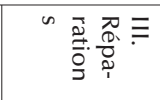 } & \multicolumn{3}{|c|}{$\begin{array}{l}\frac{1}{0}= \\
\frac{a}{w}\end{array}$} & \multicolumn{3}{|c|}{ 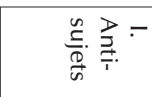 } & \multirow{2}{*}{ 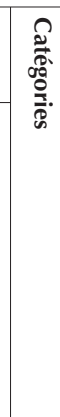 } \\
\hline & 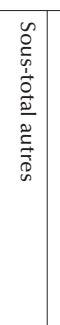 & 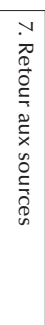 & 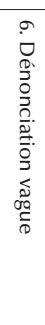 & 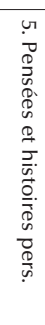 & . & 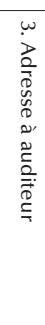 & 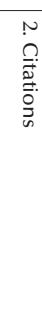 & $\overrightarrow{\vec{z}}$ & 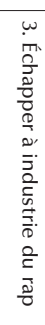 & 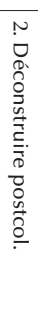 & 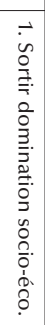 & 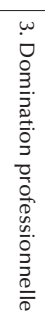 & 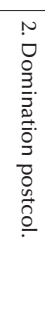 & 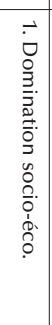 & 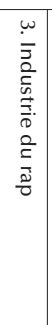 & 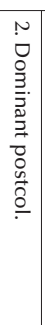 & 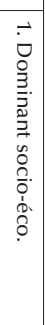 & \\
\hline $\begin{array}{l}\overrightarrow{\mathrm{o}} \\
\circ\end{array}$ & $\begin{array}{l}\text { N } \\
\stackrel{\circ}{\circ}\end{array}$ & $\begin{array}{l}\overrightarrow{\mathrm{\omega}} \\
\stackrel{\circ}{\circ}\end{array}$ & $\stackrel{\circ}{\circ}$ & $\because$ & $\therefore$ & $\therefore$ & $\therefore$ & $\vec{\circ}$ & $\vec{\circ}$ & $\stackrel{\vec{\rho}}{\circ}$ & ஓे & $\therefore$ & $\stackrel{8}{\circ}$ & $\vec{\circ}$ & $\vec{\circ}$ & : & $\therefore$ & 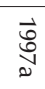 \\
\hline $\begin{array}{l}\vec{\circ} \\
\circ\end{array}$ & $\begin{array}{l}\underset{\omega}{w} \\
\stackrel{0}{\circ}\end{array}$ & $\therefore$ & $\frac{\tilde{t}}{0}$ & $\vec{\circ}$ & $\overline{0}$ & 㕝 & $\therefore$ & $\vec{\circ}$ & $\begin{array}{l}\text { wo } \\
\text { os }\end{array}$ & $\vec{\circ}$ & $\therefore$ & $\vec{\circ}$ & : & $\stackrel{\vec{\circ}}{\circ}$ & $\therefore$ & 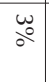 & $\stackrel{\circ}{\circ}$ & $\vec{\circ}$ \\
\hline $\overrightarrow{\mathrm{o}}$ & $\begin{array}{l}\underset{N}{w} \\
\stackrel{\circ}{\circ}\end{array}$ & $\therefore$ & $\frac{\tilde{O}}{\circ}$ & $\stackrel{w}{\circ}_{\circ}$ & 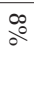 & $\therefore$ & $\therefore$ & $\vec{\circ}$ & $\stackrel{\circ}{\circ}$ & $\frac{\overrightarrow{0}}{\circ}$ & $\begin{array}{c}\overrightarrow{\omega_{0}} \\
\sigma^{\prime}\end{array}$ & 帝 & $\stackrel{\circ}{\circ}$ & $\begin{array}{c}\tilde{N} \\
\text { Oa }\end{array}$ & $\therefore$ & $\therefore$ & $\begin{array}{l}u \\
\circ \\
\circ\end{array}$ & $\begin{array}{l}\overrightarrow{\mathrm{o}} \\
\text { ه }\end{array}$ \\
\hline $\overrightarrow{\mathrm{o}}$ & $\begin{array}{c}\omega \\
\stackrel{+}{\circ} \\
\circ\end{array}$ & $\therefore$ & 응 & बे & ¿ & : & $\therefore$ & $\vec{\circ}$ & مo & $\vec{\circ}$ & 岁 & $\therefore$ & $\because$ & $\begin{array}{l}\stackrel{t}{\circ} \\
\stackrel{\circ}{\circ}\end{array}$ & $\therefore$ & $\stackrel{N}{\circ}$ & $\vec{\circ}$ & $\begin{array}{l}\overrightarrow{0} \\
\vec{\delta}\end{array}$ \\
\hline $\begin{array}{l}\overrightarrow{\mathrm{\sigma}} \\
\circ\end{array}$ & $\begin{array}{l}\vec{N} \\
\stackrel{0}{\circ}\end{array}$ & $\therefore$ & $\frac{N}{\circ}$ & $\begin{array}{l}\vec{\omega} \\
\stackrel{a}{o g}\end{array}$ & $0^{\circ}$ & $\therefore$ & $\stackrel{\omega}{\circ}$ & $\vec{\circ}$ & $\stackrel{\infty}{\circ}$ & $\vec{\circ}$ & $\stackrel{\omega}{\circ}_{\circ}$ & $\vec{\circ}$ & $\frac{\tilde{o}}{\circ}$ & $\overrightarrow{f o}$ & $\stackrel{N}{\circ}$ & $\vec{\circ}$ & $\stackrel{\infty}{\circ}$ & 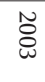 \\
\hline$\vec{\circ}$ & $\begin{array}{l}\tilde{y} \\
\text { ○े }\end{array}$ & $\therefore$ & 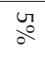 & $\vec{f}$ & $\frac{t^{\circ}}{2}$ & ֻ & $\vec{\circ}$ & $\vec{\circ}$ & \% & $\stackrel{w}{\circ}^{\circ}$ & なo & $\therefore$ & $\begin{array}{l}\tilde{N} \\
\text { o }\end{array}$ & $\begin{array}{l}\tilde{d} \\
\stackrel{0}{\circ}\end{array}$ & $\vec{\circ}$ & $\vec{\circ}$ & $\stackrel{w}{\circ}$ & 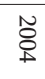 \\
\hline$\vec{\circ}$ & $\underset{\mathrm{f}}{\stackrel{\vec{o}}{\circ}}$ & $\therefore$ & जa & $\frac{\infty}{\circ}$ & $\therefore$ & $\therefore$ & $\therefore$ & $\vec{\circ}$ & ฮै & $\stackrel{2}{\circ}$ & ०。 & : & స్ & $\begin{array}{l}\omega \\
\stackrel{\omega}{\circ}\end{array}$ & $\therefore$ & a & $\stackrel{w}{\circ}$ & 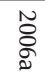 \\
\hline $\begin{array}{l}\overrightarrow{\mathrm{\sigma}} \\
\circ\end{array}$ & $\begin{array}{l}1 \\
0^{\circ}\end{array}$ & $\therefore$ & $\begin{array}{l}\omega \\
\circ\end{array}$ & $\vec{\circ}$ & $\therefore$ & $\therefore$ & \% & $\vec{\circ}$ & $\begin{array}{l}\text { w. } \\
\text { : }\end{array}$ & $\frac{t^{\circ}}{0^{2}}$ & $\stackrel{\circ}{\circ}$ & $\stackrel{\circ}{\circ}$ & 芯 & ๖े & वे & $\begin{array}{c}\omega \\
\sigma^{\circ}\end{array}$ & $\stackrel{N}{\circ}$ & 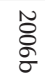 \\
\hline $\begin{array}{l}\overrightarrow{\mathrm{o}} \\
\circ \\
\circ\end{array}$ & $\begin{array}{c}\tilde{y} \\
\stackrel{0}{\circ}\end{array}$ & $\stackrel{\circ}{\circ}$ & $\frac{0}{\circ}$ & है & $\begin{array}{l}\text { : } \\
\vdots\end{array}$ & $\vec{\circ}$ & : & $\vec{\circ}$ & $\frac{\tilde{0}}{\circ}$ & $\underbrace{w}$ & $\therefore$ & $\vec{\circ}$ & $\stackrel{0}{\circ}$ & $\begin{array}{c}\tilde{\tilde{w}} \\
\tilde{o}^{\circ}\end{array}$ & ๖े & $\stackrel{0}{\circ}$ & $\begin{array}{l}w \\
:\end{array}$ & 高 \\
\hline $\begin{array}{l}\overrightarrow{\mathrm{o}} \\
\therefore\end{array}$ & $\begin{array}{c}\text { w. } \\
\text { o. } \\
\end{array}$ & $\therefore$ & 웅 & ذু & $\begin{array}{l}\bar{u} \\
\vdots \\
\vdots\end{array}$ & $\vec{\circ}$ & $\therefore$ & $\vec{\circ}$ & $\stackrel{\circ}{\circ}$ & ¿ू & $\stackrel{a}{\circ}$ & 㟧 & $\begin{array}{l}\vec{b} \\
\circ\end{array}$ & $\begin{array}{l}\vec{a} \\
{ }^{\circ}\end{array}$ & $\vec{\circ}$ & : & $\stackrel{N}{\circ}$ & స్త్ర \\
\hline ङे & 岕 & $\therefore$ & 劳 & $\stackrel{\vec{\circ}}{\circ}$ & å & $\vec{\circ}$ & $\vec{\circ}$ & $\vec{\circ}$ & ํํㅇ & ङे & है & $\therefore$ & $\vec{\circ}$ & ठু & $\therefore$ & $\stackrel{0}{\circ}$ & $\stackrel{N}{\circ}$ & : \\
\hline ठ⿳亠口冋口 & $\frac{\vec{A}}{\circ}$ & $\therefore$ & 赴 & $\begin{array}{l}\circ \\
:\end{array}$ & 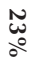 & స్ & $\stackrel{\omega}{\circ}$ & $\vec{\circ}$ & $\vec{\partial}$ & $\vec{\circ}$ & $\frac{\tilde{a}}{\partial^{\circ}}$ & $\stackrel{\omega}{\circ}$ & స్ & $\vec{\circ}$ & $\begin{array}{c}\omega \\
ٌ\end{array}$ & $\stackrel{0}{\circ}$ & $\stackrel{N}{\circ}$ & 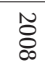 \\
\hline 훙 & $\begin{array}{c}\vec{\leftrightarrow} \\
\stackrel{\circ}{\circ}\end{array}$ & $\therefore$ & 操 & $\begin{array}{l}\tilde{0} \\
\text { : }\end{array}$ & $\begin{array}{l}\omega \\
ٌ \\
ٌ\end{array}$ & స్ & 。 & $\vec{\circ}$ & No & $\vec{\circ}$ & $\vec{\circ}$ & $\therefore$ & ठै & बुे & $\therefore$ & $\vec{\circ}$ & $\stackrel{N}{\circ}$ & స్త్రి \\
\hline$\vec{\circ}$ & $\begin{array}{l}\tilde{0} \\
\text { : } \\
0\end{array}$ & $\because$ & ֻ̃o & ळँ & $\stackrel{\vec{f}}{\circ}$ & $\stackrel{\overrightarrow{0}}{\circ}$ & $\therefore$ & $\vec{\circ}$ & $\begin{array}{l}\overrightarrow{\mathrm{w}^{\circ}} \\
\mathrm{c}^{2}\end{array}$ & : & $\begin{array}{l}\vec{\circ} \\
\stackrel{\circ}{\circ}\end{array}$ & $\vec{\circ}$ & $\stackrel{\circ}{\circ}$ & $\vec{\circ}$ & $\stackrel{N}{\circ}$ & $\stackrel{2}{\circ}$ & $\stackrel{N}{\circ}$ & 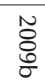 \\
\hline ङे & 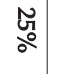 & $\therefore$ & बै & ஊ & $\vec{\circ}$ & $\vec{\circ}$ & $\therefore$ & $\vec{\circ}$ & $\stackrel{\circ}{\circ}$ & ठै & \% & $\stackrel{w}{\circ}$ & $\begin{array}{l}\text { : } \\
\circ^{\circ}\end{array}$ & $\begin{array}{l}\vec{N} \\
\stackrel{0}{\circ}\end{array}$ & $\vec{\circ}$ & $\stackrel{\omega^{\circ}}{\circ}$ & Uू & $\stackrel{\tilde{O}}{0}$ \\
\hline ذे & $\frac{\tilde{N}}{2}$ & $\therefore$ & ठै & $\stackrel{\infty}{\circ}$ & $\frac{t^{\circ}}{\sigma^{2}}$ & $\stackrel{\omega}{\circ}$ & $\therefore$ & $\vec{\circ}$ & $\begin{array}{l}\overrightarrow{\omega_{o}} \\
\sigma^{\prime}\end{array}$ & वे & $\circ^{\circ}$ & $\stackrel{N}{\circ}$ & $\begin{array}{l}\tilde{N} \\
\text { a }\end{array}$ & $\begin{array}{l}\vec{\circ} \\
\stackrel{\circ}{\circ}\end{array}$ & $\stackrel{N}{\circ}$ & $\vec{\circ}$ & जo & $\stackrel{\widetilde{O}}{=}$ \\
\hline$\vec{\circ}$ & $\begin{array}{c}\text { 崩 } \\
\vdots\end{array}$ & $\therefore$ & 虫 & $\begin{array}{l}\text { w. } \\
\text { o }\end{array}$ & ๖े & $\stackrel{N}{\circ}$ & $a^{\circ}$ & $\vec{\circ}$ & ०० & : & $\overrightarrow{\text { N }}$ & $\therefore$ & $\vec{\circ}$ & $\stackrel{\circ}{\circ}$ & $\therefore$ & $\vec{\circ}$ & $\stackrel{N}{\circ}$ & 总 \\
\hline $\overrightarrow{\mathrm{\sigma}}$ & 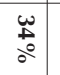 & $\vec{\circ}$ & 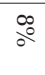 & $\begin{array}{l}\vec{\omega} \\
\stackrel{a}{o}\end{array}$ & $0^{\circ}$ & 。 & $\vec{\circ}$ & $\vec{\circ}$ & : & $\therefore$ & 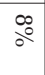 & $\vec{\circ}$ & $\vec{\sigma}$ & $\vec{a}$ & $\stackrel{N}{\circ}$ & $\stackrel{N}{\circ}$ & $\begin{array}{l}\omega \\
\stackrel{0}{\circ}\end{array}$ & $\begin{array}{l}-1 \\
\stackrel{0}{D}\end{array}$ \\
\hline
\end{tabular}


Tableau 3 : Fréquence par domination

\begin{tabular}{|l|l|}
\hline $100 \%$ & Total \\
\hline $66 \%$ & Sous-total politique \\
\hline $34 \%$ & Sous-total autres \\
\hline $28 \%$ & Sous-total socio-éco. \\
\hline $26 \%$ & Sous-total postcol. \\
\hline $12 \%$ & Sous-total industrie rap \\
\hline
\end{tabular}

Tableau 4 : Fréquence par grande catégorie

\begin{tabular}{|l|l|}
\hline $100 \%$ & Total \\
\hline $\mathbf{6 6} \%$ & Sous-total politique \\
\hline $34 \%$ & Sous-total autres \\
\hline $\mathbf{3 4} \%$ & Sous-total torts \\
\hline $\mathbf{2 6} \%$ & Sous-total réparations \\
\hline $\mathbf{7 \%}$ & Sous-total anti-sujets \\
\hline
\end{tabular}

Tableau 5 : Fréquence toutes catégories

\begin{tabular}{|l|l|}
\hline $100 \%$ & Total \\
\hline $\mathbf{1 6} \%$ & II-1. Domination socio-éco. \\
\hline $\mathbf{1 6} \%$ & II-2. Domination postcol. \\
\hline $13 \%$ & IV-5. Pensées et histoires pers. \\
\hline $\mathbf{9} \%$ & III-3. Échapper à industrie du rap \\
\hline $\mathbf{8} \%$ & III-1. Sortir domination socio-éco. \\
\hline $8 \%$ & IV-4. Égotrip \\
\hline $\mathbf{8} \%$ & III-2. Déconstruire postcol. \\
\hline $8 \%$ & 6. Dénonciation vague \\
\hline $3 \%$ & I-1. Dominant socio-éco. \\
\hline $2 \%$ & 3. Adresse à auditeur \\
\hline $\mathbf{2} \%$ & I-2. Dominant postcol. \\
\hline $\mathbf{2} \%$ & I-3. Industrie du rap \\
\hline $\mathbf{1} \%$ & II-3. Domination professionnelle \\
\hline $1 \%$ & 2. Citations \\
\hline $1 \%$ & 7. Retour aux sources \\
\hline $1 \%$ & 1. Titres \\
\hline & \\
\hline
\end{tabular}

\section{Interprétation: le rap au service des « fils d'immigrés »}

L'analyse de ces résultats montre que le R.F.I. se veut au service des « fils d'immigrés » en leur montrant l'ampleur des dominations subies, les pièges de la soumission à l'ordre dominant et les moyens de limiter ce risque.

Cette interprétation adopte l'hypothèse selon laquelle la musique « est centrale dans la compréhension de l'ordre social 52 » et son évaluation dépasse le jugement esthétique pour exprimer «nos valeurs politiques et nos idées. En ce sens, la musique participe de la manière dont nous pensons politiquement ${ }^{53}$ ». La musique, neutre par essence ${ }^{54}$, peut être utilisée politiquement : instrument de pacification (produit commercial formaté amollissant l'audience par le plaisir) ou instrument de critique (esthétique sans compromis confrontant l'audience avec le laid) ${ }^{55}$, dichotomie qui existe dans le rap dans l'opposition entre rap «commercial » validant «l'ordre dominant » et rap «conscient » le contestant. L'ordre dominant varie dans le temps et l'espace,

52. Street J., Music and politics, Cambridge, Polity, 2012, p. 6.

53. Idem, p. 159.

54. Denis-Constant, M., "Cherchez le peuple... Culture, populaire et politique », Critique internationale, 7, 2000, pp. 169-183.

55. Adorno cité dans Street J., op. cit., p. 148 ; Prévost-Thomas C., «De "Douce France" à "Tékitoi" : enjeux identitaires et sociaux de la chanson engagée en France ", in Bizzoni L., Prévost-Thomas C. (eds.), La Chanson francophone engagée, Montréal, Triptyque, 2008, p. 144 ; Traïni C., op. cit. 
qu'il soit post-ségrégationniste aux États-Unis 56 ou postcolonialiste en France ${ }^{57}$. Pour les artistes du R.F.I., l'ordre dominant est aussi professionnel, les rappeurs étant enjoints à aseptiser leur critique pour intégrer l'industrie du rap.

Se pose pourtant la question de la portée effective de la critique d'un ordre dominant. Les ambivalences entre thématiques " anti-système » et succès commercial ${ }^{58}$, tout comme la futilité de l'engagement de l'artiste comme " énervé ou révolté de service 59 », montrent l'enchevêtrement de "l'amour du pouvoir, la fascination pour le pouvoir, la crainte du pouvoir, le rejet du pouvoir 60 ». La capacité du capitalisme et de la démocratie libérale à assimiler leur propre critique soumet l'artiste engagé à l'injonction de «contestation symbolique de la réalité sociale » émanant des «fractions intellectuelles » de la classe dominante ${ }^{61}$. En réalité, la musique peut effectivement critiquer un ordre dominant par l'« action de proximité » pour « changer les comportements quotidiens » et « combattre ce qui est vécu comme une domination culturelle » par « le langage plutôt que la violence 62 ». L’interprétation des résultats montre que deux autres manières existent : l'exposition des violences symboliques de l'ordre dominant; l'exemplarité de l'artiste qui ne « se compromet » pas et prend des risques personnels. Ce travail d'exposition et ces risques personnels sont une valeur ajoutée à l'œuvre qui permet ensuite de justifier l'ascension sociale désirée et (relativement) obtenue par ces artistes.

Selon la Figure 1 63, le mot le plus fréquent est rumeur. Il apparaît lors de citations (extraits d'émissions) qui évoquent l'aspect subversif du phénomène : «Une rumeur... Information non vérifiée qui circule... Non vérifié, ça ne veut pas dire faux » (2003), «Il faut arrêter de prendre les gens qui croient les rumeurs pour des imbéciles » (idem), «Aucun contre-argument qui permette de démonter la rumeur» (2012). Le groupe s'approprie le terme de rumeur, dans le sens de « discours intrus » ouvrant un « débat légitime au sujet d'une politique de répression de la part du gouvernement français vis à vis d'une partie de sa population 64 ».

56. Martinez T., "Popular culture as oppositional culture. Rap as resistance”, Sociological perspectives, 40/2, 1997, pp. 265-286.

57. Béru L., op. cit.

58. Silvertsein P., “'What are we waiting to start the fire?’ French gangsta rap and the critique of state capitalism”, in Durand A.-P. (ed.), Black, Blanc, Beur. Rap Music and Hip-Hop Culture in the Francophone World, Lanham, The Scarecrow Press, 2002.

59. Bizzoni L., «Énonciation de la violence et violence énonciative. L’engagement dans la chanson française de la fin du XXe et début du XXIe siècle ", in Bizzoni L., Prévost-Thomas C. (eds.), La Chanson francophone engagée, Montréal, Triptyque, 2008, p. 170.

60. Denis-Constant M., op. cit., p. 177.

61. Bourdieu P., La Distinction, Paris, Éditions de Minuit, 1979, p. 334.

62. Pecqueux A., op. cit., pp. 238-240.

63. On a retiré les mots-outils et les mots polysémiques.

64. Berrada T., op. cit., pp. 63-64. 
Le deuxième mot le plus fréquent est " putain », en première position si on ajoute la variation pute(s). La grossièreté est fortement présente : gueule(s), merde, foutre, couilles, chier, etc. Ce vocabulaire contraste avec l'intellectualisme revendiqué ailleurs par Ekoué : «j’ai des diplômes à tomber par terre / Sarkozy à côté n'est qu'une vieille de-mer 65 / fais-moi confiance mon frère / tout s'accélère le monde est multipolaire » (2009b) ou «j'ai fait les mêmes écoles que tous ces hommes politiques / fait unique dans ce rap » (2008). Diplômé de Sciences Po, il conteste la violence symbolique qui suspecte d'infériorité en capital culturel les Français d'origine africaine et les banlieusards.

Ces deux séries de mots retirés, plusieurs champs lexicaux émergent. La majorité des mots les plus fréquents est connotée négativement : conflit (sang, mort, haine, guerre), détresse (sale, peur, misère) et institutions (police/flics, justice). Sont plus faiblement représentés deux regroupements plus positifs : bonheur (rêve, sourire, paix) et performance artistique (parole, plume, micro). L'ancrage dans la vie quotidienne et les rapports humains est marqué par les lieux (France/Paris/capitale, banlieue), les proches (frère, enfants, famille) et la musique.

On note que le corpus n'identifie pas certaines dominations. La dénonciation d'une domination par une religion ou par laïcisme n'apparaît jamais, la religion étant rarement mentionnée. De même, le spécisme, la destruction de l'environnement et la manipulation médiatique sont absents. De plus, l'usage d'injures à connotation sexuelle et la féminisation des antisujets ne sont pas questionnés. Sans l'encourager explicitement, le

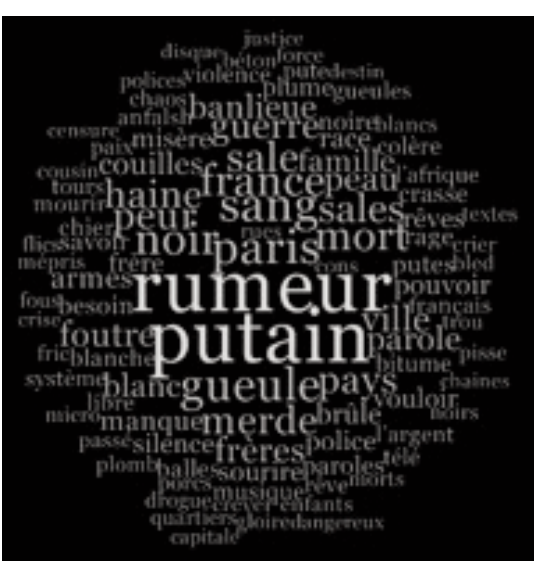

Figure 1 : Nuage des 50 mots de 4 caractères et plus les plus fréquents corpus ne dénonce pas la domination sexiste et homophobe et emploie de très nombreuses insultes à caractère sexuel. Ces thèmes apparaissent, certes rarement, dans le rap, comme chez Médine (Ni violeur ni terroriste et Al Jazeerap, sur l'islamophobie ; Combat de femme et $A$ l'ombre du mâle, sur le sexisme ; Panther blues sur le spécisme; Blokkk identitaire, sur le racisme entre Arabes et Noirs), chez Rockin Squat ou Kéry James (Démocratie fasciste et Vent d'État, sur la manipulation médiatique). Enfin, le corpus encourage explicitement, sporadiquement, certaines dominations, comme le militarisme et le nationalisme : "je rêve d'une grande Afrique forte, surarmée et souveraine » (2009b). 
Le R.F.I. utilise la musique pour critiquer un ordre qui écrase les espoirs d'une population marginalisée en raison de sa subalternité sociale, de ses origines africaines ou parce qu'elle habite en banlieue. Il revendique la légitimité pour ces populations de s'emparer selon leurs propres termes du débat public sur les différences socio-économiques et identitaires. Les références (Frantz Fanon, Aimé Césaire, Kateb Yacine) citées pour dénoncer les dominations socioéconomiques et postcolonialistes s'inscrivent dans une critique tiersmondiste classique. En outre, la diversité des origines des artistes ne se retrouve pas dans le corpus : France esclavagiste, coloniale ou postcoloniale sont traitées comme un seul et même anti-sujet opprimant de la même manière Antillais, Africains et leurs descendants présents en France.

Une décomposition approfondie confirme la centralité de l'exposition des violences symboliques et de la revendication d'exemplarité.

L'analyse des torts fait ressortir la force de la dénonciation des violences symboliques, présente dans au moins une quarantaine de chansons : "paraît qu'ici c'est le zoo, c'est le cirque, voilà l'info qui circule » (2003). Surtout lorsque le dominé est d'origine africaine : "à les écouter tous / quand l'Africain sort de sa brousse / soit il est plein de rancune / soit il en branle pas une » (idem). Le racisme scientiste passé (« espèce peu avancée / sans histoire écrite ni récit donc sans passé », 2010) comme présent (" on appelle la science qui dissèque ma race / mes rues, toits et taudis qui embarrassent », 2006a) justifie la violence physique : " on dit que je casse et agresse dans l'allégresse (...) que c'est avec le poing que la police me dresse » (2006a). Sortir de la domination postcoloniale exige de se réapproprier l'histoire : "de rafles à la benne et ce 17 octobre 61 qui croupit au fond de la Seine / on m'a demandé d'oublier » (1998). C’est également une lutte contre les clichés médiatiques : « les combats seraient à livrer en périphérie / retransmis à la télé, diffusé en différé / que T.F.1 aurait l'exclusivité du safari / présenté par une connasse d'animatrice préférée. » (2010). Mais sortir de la domination socio-économique demande plus qu'une prise de conscience, tant la démocratie libérale neutralise sa critique : «La meilleure des polices ne porte pas l'uniforme (...) La meilleure des polices / c'est ton taf, ta télé / tes crédits, tes anxiolytiques / neuroleptiques, antidépresseurs (...) la meilleure des polices / c'est tout ce qui te fait marcher droit / avec ton propre consentement » (2007b). Par ces dénonciations, le R.F.I. permet la réappropriation de la parole et la contestation du monopole des dominants à cadrer et parler d'enjeux socio-économiques et postcolonialistes.

L'analyse des réparations fait ressortir la force de la volonté d'exemplarité. Dans le sous-total industrie du rap (12\%), le contraste entre les torts (1\%, catégorie la plus faible) et les réparations (9\%) est frappant. Ce contraste est cohérent avec le fait que La Rumeur et Casey, on l'a dit, ont construit leur 
succès professionnel à l'écart des acteurs dominants de l'industrie du rap. Cela passe aussi par une rupture avec tous les acteurs de l'industrie du rap : «je revois l'ex-future star dans un bar (...) il n'a plus d'oseille et sa meuf est en cloque / il me dit qu'on devrait aller brûler son label et Skyrock / fous-moi le camp, qui t'avait dit d'aller baisser ton froc » (2006c). Cela signifie également une rupture avec les origines américaines de cette musique et ses extravagances : «Des écritures crétines / où leurs complexes cainris 66 dominent / moi, ma zone c'est l'Hexagone » (2006a). Cela signifie enfin une tension entre désir de reconnaissance et d'authenticité : « le premier disque est toujours dans les rayons / preuve d'une longévité et d'une éthique sans faille » (2008). Tension aussi vis-à-vis de l'argent : «c'est intègre que je compte abriter la famille du besoin / voilà pourquoi je me précipite pas pour toucher ma récompense » (2006b). Ces artistes, comme tout acteur engagé « en permanence soumis à l'obligation de devoir se plier à différentes normes, règles et logiques qui, parfois peuvent entrer en conflit 67 » résolvent la tension entre reconnaissance et authenticité en considérant que l'argent n'est sale que s'il est le résultat d'une soumission à «l'industrie du rap » et ses exigences : édulcoration de la critique, signature en major, passages dans les médias nationaux, etc. En résumé, le R.F.I. revendique la production d'une œuvre légitime, qualitativement supérieure à celle des « professionnels intégrés ", sans perdre, voire grâce à, leur positionnement indéfectible de «franc-tireurs ».

La leçon livrée aux «fils d'immigrés » est simple : être broyé ou récupéré par l'ordre dominant est si facile, alors souvenez-vous et faites les choses vous-mêmes. En étant connus et reconnus, commercialement et par la critique, ces artistes s'approprient et alimentent le débat public. Sur les douze modalités de contribution des dispositifs musicaux aux entreprises contestataires ${ }^{68}$, le corpus en adopte au moins sept. Par la dénonciation crue, répétée et détaillée des torts de l'ordre dominant, le corpus est susceptible de « provoquer des commotions vécues comme une prise de conscience, qui entraîne la conversion à la cause 69 ». Il active aussi un processus de communalisation en exaltant un « nous » regroupant les Français d'origine africaine et les banlieusards, donnant l'impression de "pouvoir défier le pouvoir en place ${ }^{70}$ ». Il montre explicitement du doigt un adversaire, « un "eux" désignant ceux qui profitent des injustices 71 ». Ces injustices s’inscrivent dans une filiation historique d'oppressions contre des « victimes hérö̈ques dont il conviendrait de prolonger le combat ${ }^{72}$ », d'où les références à des figures antiségrégationnistes (Angela Davis, Rosa Parks, Malcolm X) et anti-impérialistes ainsi qu'aux

\footnotetext{
66. Américains.

67. Fillieule O., op. cit., p. 207.

68. Traïi C. , op. cit., p. 60.

69. Idem, p. 22.

70. Ibid., p. 26

71. Ibid., p. 27.

72. Ibid., p. 32.
} 
Français d'origine africaine dont la mort est imputée, à tort ou à raison, à la police (17 octobre 1961, émeutes de 2005). À travers des propos exprimant explicitement « de l'exaspération, de la frustration (...) envers les processus politiques formels 73 ", le R.F.I. alimente " la critique des élus et des élites de la politique professionnelle ${ }^{74} »$. En assumant la signification du terme rumeur en tant qu' «information non vérifiée qui circule » (2003), en s'attaquant aux partis politiques de tous bords, en défendant radicalement un positionnement professionnel de franc-tireur, le R.F.I. participe à la « diffusion d'une contreinformation 75 ». Enfin, l'emploi massif d'un vocabulaire idiomatique (mélange d'argot, d'arabe et de romani) et grossier, « en érigeant des éloquences hétérodoxes en objets artistiques légitimes », fait remplir aux artistes du R.F.I. une «fonction "tribunicienne" de porte-parole les plus aptes à exprimer les préoccupations de populations en marge des institutions politiques ordinaires ${ }^{76}$ ».

Cette contribution à la contestation ne préjuge cependant pas des modalités d'engagement politique du R.F.I., qui s'inscrit bien dans la dynamique d'atomisation et de privatisation de l'engagement politique. Atomisation car le rejet des élites politiques, économiques et culturelles est massif et intransigeant. Elle est particulièrement marquée avec le rejet des autres rappeurs, même « conscients » : «Demande à Abd al Malik de te chanter la Marseillaise / en voilà un bon lèche-cul entre parenthèses » (2009b). Privatisation car audelà d'une dénonciation globale, le R.F.I. revendique des engagements effectifs qui sont précis et limités : absence de promotion, voire rejet, des formes d'engagement traditionnels (vote, syndicalisme, action associative, etc.), dénonciation des violences symboliques de l'ordre dominant, exemplarité professionnelle. L'ambivalence vis-à-vis de la réussite professionnelle souligne à la fois la persistance des préoccupations « matérialistes 77 » dans l'engagement politique contemporain et la difficulté de rester " authentique ", défi constant des rappeurs : « le poste d'interprétation particulier qu'ils s'imposent (auteur / interprète / protagoniste / adresse systématique), impliquant fidélité à soi et aux autres inédite dans l'histoire de la chanson 78 ». De la sorte, le corpus reflète bien l'émergence de ce « citoyen moins respectueux des pouvoirs institués et des élites ", qui n’hésite pas à "explorer les voies de l'engagement protestataire », en un mot « ce citoyen inquiet et imprévisible d'une démocratie toujours conflictuelle 79 ».

73. Hay C., op. cit., p. 75.

74. Traïni C., op. cit., p. 38.

75. Idem, p. 60.

76. Ibid., p. 48.

77. Mathieu L., op. cit., pp. 29-31.

78. Pecqueux A., Voix du rap, op. cit., p. 240.

79. Perrineau P., op. cit., p. 117. 
L'objectif d'examiner les modalités politiques du R.F.I. fait ressortir la critique d'un ordre dominant à trois dimensions : domination socio-économique, postcolonialiste et professionnelle. Le R.F.I. critique effectivement un ordre dominant de deux manières : par l'exposition des violences symboliques de l'ordre dominant et par l'exemplarité démontrée par les artistes, qui ont pris des risques (financiers et judiciaires) personnels. En rejetant les élites et en prônant une lutte concrète, limitée et individuelle, le R.F.I. s'insère dans la dynamique contemporaine d'atomisation et de privatisation de l'engagement politique.

Il conviendrait de compléter cette analyse par l'examen des dominations ignorées ou encouragées par le corpus (religion, patriarcat hétéro-normé, etc.), en tenant compte de l'évolution du contenu dans le temps et en analysant d'autres rappeurs politiques.

En contrepoint de la «production institutionnelle de l'illégitimité du genre, une illégitimité non seulement culturelle, mais aussi politique », caractérisée par les offensives du gouvernement et du parlement français contre plusieurs rappeurs, La Rumeur et Casey participent de la réappropriation par les rappeurs de la « définition publique » du rap, ayant longtemps assigné les rappeurs au minoritaire et « leurs pratiques aux banlieues ", pour en faire "un moyen d'existence publique relayant des expériences, des sentiments, des points de vue qui n'avaient guère ou pas droit de cité auparavant 80 ». Casey elle-même ne dit pas autre chose : "Quelle aubaine pour une peau d'ébène d'accéder à la scène / quelle veine d'y voir sa parole souveraine anciennement souterraine / sans censure ou larsen de la clique politicienne (...) la gent parisienne peut être mondaine, babylonienne / contrôler l'antenne télé, la bande $\mathrm{FM} /$ je sais que mon emblème, ma cause avec haine lui pose problème. » (2006a).

En ce sens, le rap politique n'est pas un objet à part dans l'art engagé. Au contraire, il assimile et répond aux contraintes matérielles et idéologiques de la production artistique engagée contemporaine, en particulier le désenchantement envers les grandes causes. Le rap (politique) n'est ainsi qu'une autre forme d'art (politique). Sans doute doit-on y voir « l'un des plus riches héritiers 81 » de la chanson française engagée ${ }^{82}$. Une filiation revendiquée par Ekoué : " pourquoi on dérogerait à l'art du guignol, à la tradition française du brocard du politique de façon salace, gratuite, vulgaire, méchante 83 ? ».

80. Hammou K., op. cit., p. 258.

81. Rubin C., "Le rap est-il soluble dans la chanson française ?", Volume, 3/2, 2004, p. 40.

82. Prévost-Thomas C., op. cit., pp. 144-145.

83. Offner F., "La Rumeur, tenaces du rap : “On est dans une 'démocrature”" », Rue 89, 17 septembre 2009 (http://blogs.rue89.nouvelobs.com/actu-rap/2009/11/17/la-rumeur-tenaces-durap-on-est-dans-unedemocrature, Page consultée le 2 juin 2015). 
Héritier mais aussi prolongateur, car il propose une autocritique de la gestion de l'altérité de la société française. Or l'autocritique est ce « qui entrave, énerve, bloque, mais qui permet aussi d'éviter de prendre des messies pour des lanternes. (...) [On perçoit que] notre époque est celle de la fin des idéologies et que, ce faisant, elle a fait un pas de plus vers une maturité... encore bien lointaine ${ }^{84}$ ». Dans cette perspective, les critiques du « rap de fils d'immigrés » peuvent être comprises comme une contribution, parfois outrancière ou violente mais salutaire, aux débats publics sur la place des Français d'origine africaine dans ce pays.

\section{Discographie}

1997 : La Rumeur, Premier Volet : le Poison d'avril, FUAS Music, 1997.

1998 : La Rumeur, Deuxième volet : le Franc-Tireur, FUAS Music, 1998.

1999a : La Rumeur, L'Entre-volet, FUAS Music.

1999b : La Rumeur, Troisième volet : Le Bavar et le Paria, FUAS Music.

2003 : La Rumeur, L'Ombre sur la mesure, EMI.

2004 : La Rumeur, Regain de tension, La Rumeur Records.

2006a : Casey, Ennemi de l'ordre, Anfalsh Productions et Dooeen Damage.

2006b : Casey, Hostile au stylo, Anfalsh Productions et Dooeen Damage.

2006c : Casey, Tragédie d'une trajectoire, Anfalsh Productions. 2007a : La Rumeur, 1997-2007, les inédits, La Rumeur Records. 2007b : La Rumeur, Du cœur à l'outrage, La Rumeur Records. 2008 : Ekoué, Nord Sud Est Ouest premier épisode, La Rumeur Records.

2009a : Casey (avec Hamé et Zone libre), L'Angle mort, T-Rec.

2009b : Ekoué et Le Bavar, Nord Sud Est Ouest deuxième épisode, La Rumeur Records.

2010 : Casey, Libérez la bête, Ladilafé et Anfalsh Productions. 2011 : Casey (avec B. James et Zone libre), Les Contes du chaos, Intervalle Triton.

2012 : La Rumeur, Tout brûle déjà, La Rumeur Records.

84. Tavoillot P.-H., « Les idéologies après la fin des idéologies », Les Cabiers français, 364, 2011, p. 20. 